\title{
On the Invariance of the Set of Core Matchings with Respect to Preference Profiles*
}

\author{
Ruth Martínez $^{\dagger}$, Jordi Massó ${ }^{\ddagger}$, Alejandro Neme ${ }^{\dagger}$, and Jorge Oviedo ${ }^{\dagger}$
}

July 2009

\begin{abstract}
We consider the general many-to-one matching model with ordinal preferences and give a procedure to partition the set of preference profiles into subsets with the property that all preference profiles in the same subset have the same Core. We also show how to identify a profile of (incomplete) binary relations containing the minimal information needed to generate as strict extensions all the (complete) preference profiles with the same Core. This is important for applications since it reduces the amount of information that agents have to reveal about their preference relations to centralized Core matching mechanisms; moreover, this reduction is maximal.
\end{abstract}

Keywords: Matching, Core.

Journal of Economic Literature Classification Number: C78.

${ }^{*}$ We are very grateful to Flip Klijn for his helpful comments. We also thank members of the Bag Lunch Workshop on Game Theory and Social Choice at Universitat Autònoma de Barcelona for comments and suggestions. The work of R. Martínez, A. Neme, and J. Oviedo is partially supported by the Universidad Nacional de San Luis through grant 319502 and by the Consejo Nacional de Investigaciones Científicas y Técnicas through grant PICT-02114. Support for the research of J. Massó was received through the prize "ICREA Acadèmia" for excellence in research, funded by the Generalitat de Catalunya. He also acknowledges the support of the Barcelona Graduate School of Economics, where he is an affiliated professor, through its Research Recognition Programme. His work is also supported by the Spanish Ministry of Science and Innovation through grants ECO2008-04756 (Grupo Consolidado-C) and CONSOLIDER-INGENIO 2010 (CDS2006-00016), and by the Generalitat de Catalunya through grant SGR2009-419.

${ }^{\dagger}$ Instituto de Matemática Aplicada de San Luis. Universidad Nacional de San Luis and CONICET. Ejército de los Andes 950. 5700, San Luis, Argentina. E-mails: martinez@unsl.edu.ar, aneme@unsl.edu.ar, and joviedo@unsl.edu.ar

†'Departament d'Economia i d'Història Econòmica and CODE. Universitat Autònoma de Barcelona. 08193, Bellaterra (Barcelona), Spain. E-mail: jordi.masso@uab.es 


\section{Introduction}

Consider a set of agents and a set of allocations over which each agent has a preference relation. Suppose an allocation is proposed. A coalition (a subset) of agents blocks the allocation if all its members can improve upon the allocation by seceding and remaining at least as well as they were at the proposed allocation and at least one of them being strictly better off. The Core is the set of unblocked allocations. The Core and the Shapley value have been the most undisputed and used solution concepts for cooperative games. The Core emphasizes the stability requirements of the social institution that has to propose allocations as potential solutions of the problem.

The purpose of this paper is to contribute to a better understanding of the Core for ordinal, two-sided and many-to-one matching problems. ${ }^{1}$ A matching problem consists of two non-empty and disjoint sets of agents: the set of firms (or institutions like schools, colleges, hospitals, etc.) and the set of workers (or individuals like children, students, medical interns, etc.). An allocation for a matching problem is a matching among firms and workers with the property that each worker can be matched to at most one firm and each firm is matched to a subset (possibly empty) of workers, keeping the bilateral nature of the relationships in the sense that if a worker is matched to a firm this firm is matched to a subset of workers that contains this worker. Each worker has a (strict) preference relation on the set of firms plus the prospect of remaining unmatched. Each firm has a (strict) preference relation on the set of all subsets of workers. A preference profile is a list of preference relations, one for each agent. The Core of a matching problem (at a given preference profile) is the set of matchings that are not blocked; namely, a matching is in the Core if there is no subset of agents (a coalition of firms and workers) such that, by rematching only among themselves, each agent gets a weakly better partner and at least one of them gets a strictly better one. There is a large literature studying the Core of matching problems (and related models) as well as the strategic incentives induced to agents by direct revelation mechanisms that select a Core matching for each declared preference profile. ${ }^{2}$

\footnotetext{
${ }^{1}$ From now on we will refer to an instance of those as a matching problem. See Echenique and Oviedo (2004) for an analysis of the Core of any matching problem using Tarki's Fixed Point Theorem.

${ }^{2}$ See Roth and Sotomayor (1990) for an illuminating and comprehensive survey of this literature as well as for an exhaustive bibliography that Al Roth maintains updated in his webside
} 
The first result of the paper characterizes the family of equivalence classes of preference relations of each firm with the property that two preference relations are in the same class if and only if they have the same Core for all preference relations of the remaining agents. Thus, it shows how to partition the set of preference profiles into subsets with the property that all preference profiles in the same subset have the same Core. Specifically, take a preference profile and a firm. Consider two subsets of workers $S$ and $S^{\prime}$. Suppose that the firm prefers $S$ to $S^{\prime}$. Replace in the original preference profile the preference relation of the firm with another one in which now the firm prefers $S^{\prime}$ to $S$ and all other orderings remain unchanged. Depending on the original preference profile, the selected firm and the two subsets of workers $S$ and $S^{\prime}$, the Core may either change or remain the same. Our invariance result (Theorem 1) identifies those orderings between pairs of subsets of workers in a preference relation of a firm that, if inverted, the Core remains unchanged for all possible preference relations of the other agents. In other words, Theorem 1 identifies irrelevant changes on a preference relation of a firm that leave the Core invariant, irrespectively of the other agents' preference relations. The way of proceeding with this identification is as follows. Note first that a preference relation of a firm orders all subsets of workers (i.e., it is a complete binary relation on the power set of the set of workers). Build a binary relation by first constructing a family of subsets of workers with the property that a set is in the family if and only if the firm, if confronted with this set of workers, considers that the set itself is the best among all of its subsets according to the firm's (complete and original) preference relation; equivalently, erase from the power set of the set of workers those subsets that, if offered to the firm, would not be chosen because the firm would like to fire some of its workers. We call this collection of subsets of workers the family of individually rational subsets of workers relative to the firm's preference relation. Second we define a binary relation on this family as follows: given two subsets of workers $S$ and $S^{\prime}$ in the family we declare that $S$ is preferred to $S^{\prime}$ (according to the binary relation) if and only if $S$ is the best subset (according to the original and complete preference relation of the firm) among all subsets of $S \cup S^{\prime}$; otherwise, the subsets are left unordered by the binary relation. Observe that in general this binary relation is incomplete and only defined on a subfamily of subsets of workers. It turns out that this binary relation inherits some (but http://kuznets.fas.harvard.edu/ aroth/alroth.html. 
not all $)^{3}$ properties of the original preference relation and can be used as the representative of one equivalence class because all preference relations of the firm that share the same binary relation constructed as we just described have the property that the Core is the same regardless of the other agents' preference relations. Note that, given a preference relation of a worker, we could similarly construct its corresponding binary relation on the set of acceptable firms. However, this binary relation on the set of acceptable firms coincides with the initial complete preference relation (on the set of acceptable firms) since the best firm of the union of two different firms is always equal the best of the two firms. Thus, from the point of view of the workers' preference relations all orderings (between pairs of acceptable firms) are relevant for the set of Core matchings. This is the reason why preference relations of workers will remain fixed while we identify equivalence classes of preferences of firms.

Theorem 1 extends and generalizes our previous result in Martínez, Massó, Neme, and Oviedo (2008) where we construct this invariant partition only for the subclass of substitutable preference profiles. ${ }^{4}$ In this case, the Core and the set of stable matchings coincide, are non-empty, and the binary relations obtained from the preference relations of the firms, as we have described above, have more structure. They are individually rational, ordered, and closed semilattices. ${ }^{5}$

\footnotetext{
${ }^{3}$ It is a reflexive, antisymmetric, and acyclic binary relation that has a maximal element on the family of individually rational subsets of workers. In general, it is not transitive.

${ }^{4} \mathrm{~A}$ preference relation of a firm is substitutable if the desirability of a worker $w$ in a particular set of workers does not come from the presence of another worker $w^{\prime}$ in that set because the firm still wants to hire worker $w$ even when worker $w^{\prime}$ is not available anymore; i.e., substitutable preference relations do not exhibit strong complementarities among workers. A preference profile is substitutable if the preference relations of all firms are substitutable. Kelso and Crawford (1982) were the first to define and use substitutability in a more general matching model with money.

${ }^{5} \mathrm{~A}$ semilattice is a partially ordered set with the property that the least upper bound of any pair of elements in the set exists. A semilattice is individually rational if the partially ordered family of subsets of workers is composed of those sets that are preferred to their subsets. A semilattice is ordered if for all subsets $S$ and $S^{\prime}$ in the partially ordered family of subsets of workers, the least upper bound (according to the partial order) of $S$ and $S^{\prime}$ coincides with the least upper bound (according to the partial order) of all subsets of $S \cup S^{\prime}$, and in addition, this set is contained in $S \cup S^{\prime}$. A semilattice is closed if all subsets of each set in the family are themselves elements of the family.
} 
In general, the binary relation used to represent the equivalence class formed by all preference relations of a firm that leave the Core invariant still relates too many pairs of subsets of workers. In centralized matching markets in which Core mechanisms (stable ones, whenever firms' preferences are substitutable) are used to suggest to the participants - after collecting and processing their preference relations- a matching in the Core, it would be very useful to use the smallest possible amount of information contained in the preference profile that still allows to compute a Core matching relative to this preference profile. ${ }^{6}$ Thus, and in order to identify this minimal amount of information, we give a procedure to construct the minimal binary relation contained in the binary relation identified in Theorem 1 , with the property that it still can generate all preference relations in the same equivalence class (that is, with the same Core) as their strict extensions. Furthermore, this binary relation is minimal in the sense that any strictly weaker (i.e., strictly contained) binary relation has at least two strict extensions that belong to different equivalence classes and thus have different Cores for some preference relations of the other agents. We say that a preference relation (over the set of all subsets of workers) is a strict extension of a binary relation (on the family of individually rational subsets of workers) if (1) the preference relation agrees on all pairs already related by the binary relation, (2) a set in the family is declared as strictly preferred by the extension to all of its strict subsets, and (3) any set that does not belong to the family has a strict subset in the family that is strictly preferred by the extension.

Observe that the question of finding the minimal binary relation that can generate all equivalent preference relations was not even asked in Martínez, Massó, Neme, and Oviedo (2008) for the subclass of substitutable preference relations. Thus, the marginal contribution of this paper in relation to our former one is two-fold. We first extend the result of Theorem 1 from substitutable preference profiles to any preference profile. Second, we identify for each preference relation (substitutable or not) the minimal binary relation that can be used as the representative of each equivalence class of preference relations with an invariant Core. This binary relation contains the indispensable and, at the same time,

\footnotetext{
${ }^{6}$ Again, see Roth and Sotomayor (1990) for a general description and analysis of these centralized markets. Niederle, Roth, and Sönmez (2008) contains a recent overview on matching and market design in general.
} 
minimal information to generate the full class.

Echenique (2008) answers a related question. Suppose we observe a set of matchings and we do not know agents' preference relations. Are there preference relations for the agents so that the observed set of matchings are stable? If yes, the set of matchings is said to be rationalizable. Echenique (2008) first shows that there are sets of matchings that are not rationalizable (and thus, the theory is testable) and second he identifies conditions that characterize the sets of matchings that are rationalizable: a necessary condition is that a certain graph has no odd cycles and a necessary and sufficient condition is in terms of no odd cycles and a certain system of polynomial inequalities. However, his results are different from ours in many respects. Echenique's results apply only to the one-to-one matching model while ours apply to the more general many-to-one matching model. His results are in graph-theoretical terms and deal with the full preference profile by identifying how agents can rank potential partners given the set of matchings to be rationalizable. In contrast we identify, given a preference relation of a firm over subsets of workers (and independently of the other agents' preferences), those relations between pairs of subsets of workers that are critical from the point of view of the Core and those that are not.

Before finishing this Introduction we want to emphasize that, besides their intrinsic interest, our invariance and minimality results have also at least three different type of relevant implications. (1) Informational: our results show that the amount of information about firms' preferences required to compute the set of Core matchings may be significantly smaller than the amount needed to describe their complete preference relations. This may be specially relevant for running direct preference revelation Core mechanisms in centralized entry-level professional labor markets. (2) Computational: our results may simplify the task of computing the set of Core matchings. They show that for this purpose it suffices to compute the Core at those profiles for which firms' complete preference relations are replaced by their corresponding minimal binary relations. For instance, suppose we know the Core at a given profile. Consider another preference profile whose firms' preference relations induce the same profile of minimal binary relations than the former one. Then our results say that the Core at the two profiles coincide. (3) Behavioral: our results simplify the analysis of the strategic behavior induced on firms by centralized Core matching mechanisms. In particular, to find either best-replies or unilateral deviations may be 
substantially easier.

The paper is organized as follows. In Section 2 we present the notation, the basic definitions, and some preliminary results. In Section 3 we state and prove the invariance result for the set of Core matchings. In Section 4 we define the notions of minimal binary relation, strict extension and state and prove the minimality result. Finally, in Section 5 we conclude with a general description of the procedure that partitions the set of preference profiles.

\section{Preliminaries}

\subsection{Agents and Preferences}

Let $W$ be the set of workers and let $F$ be the set of firms. We assume that $W$ and $F$ are finite and disjoint. The set of agents is $W \cup F$. Each worker $w \in W$ has a (strict) preference relation $P_{w}$ over the set of firms plus the prospect of remaining unemployed. Specifically, we assume that $P_{w}$ is a complete, irreflexive, and transitive binary relation on $F \cup\{\varnothing\}$, where $\varnothing$ means that $w$ is not hired by any firm. ${ }^{7}$ Given $P_{w}$, let $R_{w}$ be the weak preference relation over $F \cup\{\varnothing\}$ induced by $P_{w}$ as follows: for $f, f^{\prime} \in F \cup\{\varnothing\}, f R_{w} f^{\prime}$ if and only if $f^{\prime} P_{w} f$ does not hold. Then, $R_{w}$ is a complete, reflexive, antisymmetric, and transitive binary relation on $F \cup\{\varnothing\} .^{8}$ Each firm $f \in F$ has a (strict) preference relation $P_{f}$ over the family of all subsets of workers. Specifically, we assume that $P_{f}$ is a complete, irreflexive, and transitive binary relation on $2^{W}$, where the empty set is interpreted as the prospect of not hiring any worker. Given firm $f$ 's preference relation $P_{f}$ and a subset of workers $S, C h\left(S, P_{f}\right)$ denotes $f$ 's most-preferred subset of $S$ according to $P_{f}$. Generically, we will refer to this set as the choice set. Given $P_{f}$, let $R_{f}$ be the complete, reflexive, antisymmetric, and transitive binary relation induced similarly on $2^{W}$ by $P_{f}$. A preference profile $P=\left(\left(P_{f}\right)_{f \in F},\left(P_{w}\right)_{w \in W}\right)$ is a $|F|+|W|$-tuple of preference relations, one for each agent. Given a preference profile $P$ and $f$ 's preference relation $P_{f}^{\prime}$, we will denote by $\left(P_{f}^{\prime}, P_{-f}\right)$ the original preference profile

\footnotetext{
${ }^{7}$ A binary relation $\succ$ on $X$ is complete if for all $x, y \in X$ such that $x \neq y$, either $x \succ y$ or $y \succ x$; irreflexive if $x \nsucc x$ for all $x \in X$; and transitive if for all $x, y, z \in X$ such that $x \succ y \succ z, x \succ z$ holds.

${ }^{8} \mathrm{~A}$ binary relation $\succeq$ on $X$ is reflexive if $x \succeq x$ for all $x \in X$; and antisymmetric if, for all $x, y \in X$ such that $x \succeq y$ and $y \succeq x, x=y$ holds.
} 
$P$ after replacing $P_{f}$ by $P_{f}^{\prime}$ and refer to $P_{-f}$ as a subprofile. Given a preference relation $P_{f}$ of firm $f$, the subsets of workers preferred to the empty set by $f$ are called acceptable. Similarly, given a preference relation $P_{w}$ of worker $w$, the firms preferred to the empty set by $w$ are called acceptable. By convention, we declare the empty set as being acceptable for all agents. Since the set of agents will be fixed throughout the paper, we identify a matching problem with a preference profile $P$.

\subsection{Matchings}

A matching assigns each firm to a subset of workers (possibly empty) and each worker to at most one firm, keeping the bilateral nature of the relationship; i.e., worker $w$ works for firm $f$ if and only if firm $f$ hires worker $w$.

Definition 1 A matching is a mapping $\mu: W \cup F \longrightarrow 2^{F \cup W}$ with the properties:

(ma.1) $\mu(f) \in 2^{W}$ for all $f \in F$;

(ma.2) $\mu(w) \in 2^{F}$ and $|\mu(w)| \leq 1$ for all $w \in W$; and

(ma.3) $w \in \mu(f)$ if and only if $\mu(w)=f .^{9}$

We follow the convention of describing matchings by a table. For instance, given $W=$ $\left\{w_{1}, w_{2}, w_{3}, w_{4}, w_{5}\right\}$ and $F=\left\{f_{1}, f_{2}, f_{3}\right\}$, the table

\begin{tabular}{c|ccc|}
\multicolumn{1}{c}{} & $f_{1}$ & $f_{2}$ & $f_{3}$ \\
\cline { 2 - 4 }$\mu$ & $\left\{w_{1}, w_{2}\right\}$ & $\left\{w_{3}, w_{4}\right\}$ & $\varnothing$ \\
$\mu^{\prime}$ & $\left\{w_{2}, w_{5}\right\}$ & $\left\{w_{3}\right\}$ & $\left\{w_{1}, w_{4}\right\}$ \\
\cline { 2 - 4 } & &
\end{tabular}

means that at $\mu, f_{1}$ is matched to $w_{1}$ and $w_{2}, f_{2}$ is matched to $w_{3}$ and $w_{4}$, and $f_{3}$ and $w_{5}$ are unmatched. Similarly, at $\mu^{\prime}, f_{1}$ is matched to $w_{2}$ and $w_{5}, f_{2}$ is matched to $w_{3}$, and $f_{3}$ is matched to $w_{1}$ and $w_{4}$.

\subsection{The Core}

If matching is voluntary it should be immune to any secession of a coalition of agents that, by matching only amongst themselves, could obtain better partners by breaking the former

\footnotetext{
${ }^{9}$ With a slight abuse of notation we treat $\mu(w) \neq \varnothing$ as an element of $F$ instead of one of its subsets; for instance, we write $\mu(w)=f$ instead of $\mu(w)=\{f\}$.
} 
partnerships and creating new ones (a block). The Core is the set of matchings that are not blocked by any coalition of agents.

Definition 2 Let $P$ be a preference profile and let $\mu$ be a matching. Coalition $W^{\prime} \cup F^{\prime} \subseteq$ $W \cup F$ blocks $\mu$ if there exists another matching $\mu^{\prime}$ such that:

(bl.1) $\mu^{\prime}(f) \subseteq W^{\prime}$ for all $f \in F^{\prime}$;

(bl.2) either $\mu^{\prime}(w)=\varnothing$ or $\mu^{\prime}(w) \in F^{\prime}$ for all $w \in W^{\prime}$; and

(bl.3) for all $f \in F^{\prime}$,

$$
\mu^{\prime}(f) R_{f} \mu(f),
$$

for all $w \in W^{\prime}$,

$$
\mu^{\prime}(w) R_{w} \mu(w)
$$

and at least one of the weak preferences in (1) and (2) is strict.

Definition 3 Let $P$ be a preference profile. A matching $\mu$ is in the Core (at $P$ ) if it is not blocked by any coalition.

A matching $\mu$ is individually rational (at $P$ ) if $\mu(w) R_{w} \varnothing$ for all $w \in W$ and $\mu(f)=$ $C h\left(\mu(f), P_{f}\right)$ for all $f \in F$. Denote by $I R(P)$ the set of individually rational matchings at $P$. A matching $\mu$ is pair-wise stable (at $P$ ) if there is no unmatched pair $(w, f) \in W \times F$ such that $f P_{w} \mu(w)$ and $w \in C h\left(\mu(f) \cup\{w\}, P_{f}\right)$. The set of stable matchings (at $P$ ) is the set of individually rational matchings that are pair-wise stable. Let $S(P)$ denote the set of stable matchings (at $P$ ) and let $C(P)$ denote the set of matchings in the Core (at $P)$. Obviously, $C(P) \subseteq S(P)$ for all $P$. It is well-known that there are preference profiles for which the Core (and the set of stable matchings) is empty. Example 1 below exhibits a preference profile with an empty Core.

Example 1 Let $F=\left\{f_{1}, f_{2}\right\}$ be the set of firms and $W=\left\{w_{1}, w_{2}\right\}$ be the set of workers. Consider the preference profile $P=\left(P_{f_{1}}, P_{f_{2}}, P_{w_{1}}, P_{w_{2}}\right)$

$$
\begin{array}{cccc}
\frac{P_{f_{1}}}{\left\{w_{1}, w_{2}\right\}} & \frac{P_{f_{2}}}{\left\{w_{2}\right\}} & \frac{P_{w_{1}}}{f_{2}} & \frac{P_{w_{2}}}{f_{1}} \\
\left\{w_{1}\right\} & \left\{w_{1}\right\} & f_{1} & f_{2} \\
\varnothing & \varnothing & \varnothing & \varnothing
\end{array}
$$


where we only list acceptable partners in decreasing order. ${ }^{10}$ The table below identifies a blocking coalition for each individually rational matching.

\begin{tabular}{c|ccc|}
\multicolumn{1}{c}{} & $f_{1}$ & $f_{2}$ & a blocking coalition \\
\cline { 2 - 4 }$\mu_{1}$ & $\left\{w_{1}, w_{2}\right\}$ & $\varnothing$ & $\left\{w_{1}, f_{2}\right\}$ \\
$\mu_{2}$ & $\left\{w_{1}\right\}$ & $\varnothing$ & $\left\{w_{2}, f_{2}\right\}$ \\
$\mu_{3}$ & $\left\{w_{1}\right\}$ & $\left\{w_{2}\right\}$ & $\left\{w_{1}, w_{2}, f_{1}\right\}$ \\
$\mu_{4}$ & $\varnothing$ & $\varnothing$ & $\left\{w_{1}, f_{1}\right\}$ \\
$\mu_{5}$ & $\varnothing$ & $\left\{w_{1}\right\}$ & $\left\{w_{2}, f_{2}\right\}$ \\
$\mu_{6}$ & $\varnothing$ & $\left\{w_{2}\right\}$ & $\left\{w_{1}, f_{1}\right\}$ \\
\hline
\end{tabular}

Thus, $C(P)=\varnothing$.

Kelso and Crawford (1982) proposed (in a more general many-to-one matching model) a condition on the preference relations of firms, called substitutability, with the property that if in a profile $P$ all firms have substitutable preference relations then the Core is non-empty and coincides with the set of stable matchings. For this reason substitutability has played a central role in the analysis of many-to-one matching models.

Definition 4 A firm $f$ 's preference relation $P_{f}$ satisfies substitutability if for any set $S$ containing workers $w$ and $w^{\prime}\left(w \neq w^{\prime}\right)$, if $w \in C h\left(S, P_{f}\right)$ then $w \in C h\left(S \backslash\left\{w^{\prime}\right\}, P_{f}\right)$.

Substitutability precludes strong complementarities among workers since it requires that the desirability of a worker $w$ in a particular set $S$ does not come exclusively from the presence of another worker $w^{\prime}$ in that set; i.e., the firm still wants to hire worker $w$ even though worker $w^{\prime}$ is not available anymore; thus, $w$ is a good worker (in the context of the set $S$ ) not only because of the presence of $w^{\prime}$. A preference profile $P$ is substitutable if for each firm $f$, the preference relation $P_{f}$ satisfies substitutability. Let $\mathcal{S}$ be the set of substitutable preference profiles. For any substitutable preference profile $P \in \mathcal{S}, C(P)=S(P) \neq \varnothing$. However, there are non substitutable preference profiles $P$ for which $C(P) \neq \varnothing$. Observe that this statement does not contradict Hatfield and Kojima's (2008) result stating that the set of substitutable preference profiles $\mathcal{S}$ is the maximal domain of preference profiles

\footnotetext{
${ }^{10}$ Observe that in general the relative orderings of unacceptable partners (subsets of workers or individual firms) are irrelevant from the point of view of the set of matchings in the Core since any agent can block any matching in which he is matched to an unacceptable partner.
} 
containing $\mathcal{S}$ under which the set of Core matchings is non-empty. ${ }^{11}$ In their setting, maximal domain means that if $P_{f}$ is not substitutable then there exists a substitutable subprofile $P_{-f}$ (perhaps a unique one) with the property that $C\left(P_{f}, P_{-f}\right)=\varnothing$. However, in general there may exist many subprofiles $P_{-f}^{\prime}$ for which $C\left(P_{f}, P_{-f}^{\prime}\right) \neq \varnothing$.

\subsection{Extracting Binary Relations from Firms' Preferences}

To identify, given a preference relation of a firm (over the family of all subsets of workers), the subfamily of subsets of workers on which we will define the partial order that keeps only the relevant relations (from the point of view of the Core) between subsets of workers, we need some additional notions and notation.

Let $A$ be a non-empty subfamily of subsets of $W$ containing the empty set; i.e., $A \subseteq 2^{W}$ and $\varnothing \in A$. A partial order $\succeq$ on $A$ is a reflexive, transitive, and antisymmetric binary relation on $A$. Observe that weak preferences of firms are complete partial orders on $2^{W}$. Given a binary relation $\succeq$ on $A$, let $\succ$ be the antireflexive and transitive binary relation on $A$ induced by $\succeq$ on $A$ as follows: for $S, S^{\prime} \in A, S \succ S^{\prime}$ if $S \succeq S^{\prime}$ and $S \neq S^{\prime}$. A binary relation $\succeq$ on $A$ is acyclic if for all $S_{1}, \ldots, S_{k} \in A$ such that $S_{1} \neq S_{k}, S_{1} \succeq \ldots \succeq S_{k}$ implies $S_{k} \nsucceq S_{1}$. A binary relation $\succeq$ on $A$ has a maximal element on $B \subseteq A$ if there exists $S \in B$ such that for all $S^{\prime} \in B$ with $S^{\prime} \neq S, S \succ S^{\prime}$ holds where $\succ$ is induced by $\succeq$. Then, given a preference relation $P_{f}$ and a set $S \in 2^{W}, P_{f}$ has a maximal element on the family of all subsets of $S$. We have denoted this set by $C h\left(S, P_{f}\right)$ and called it the choice set of $S$ according to $P_{f}$; namely, $C h\left(S, P_{f}\right) P_{f} S^{\prime}$ for all $S^{\prime} \in 2^{S} \backslash C h\left(S, P_{f}\right)$.

It will be useful to understand (and to denote) a binary relation $\succeq$ on $A$ as a subset of $A \times A$; namely, for all $S, T \in A,(S, T) \in \succeq \subseteq A \times A$ if and only if $S \succeq T$. Hence, for two binary relations $\succeq$ and $\succeq^{\prime}$ on $A$ the notation $\succeq \subseteq \succeq^{\prime}$ means that if $S, S^{\prime} \in A$ and $S \succeq S^{\prime}$ then, $S \succeq^{\prime} S^{\prime}$.

After these preliminaries we now turn to define the procedure to delete from the preference relation of a firm the orderings between those pairs of subsets of workers that are

\footnotetext{
${ }^{11}$ In fact, Hatfield and Kojima (2008) shows that this maximality result holds for the set of weak substitutable preference profiles in the more general model of matching with contracts. Their weak substitutability condition in the model of matching with contracts coincides with the notion of substitutability in the purely ordinal matching model analyzed in this paper.
} 
irrelevant with respect to the set of matchings in the Core. First, subsets that are not the choice set of themselves can be left unordered since no matching in the Core, regardless of the other agents' preference relations, matches this firm with any of these subsets. Formally, given the preference relation $P_{f}$ on $2^{W}$, define the family $A_{P_{f}}$ of individually rational subsets of workers relative to $P_{f}$ as the collection of sets that are choice sets of themselves; that is,

$$
A_{P_{f}}=\left\{S \in 2^{W} \mid S=C h\left(S, P_{f}\right)\right\} .
$$

Second, some pairs of subsets of workers in $A_{P_{f}}$ will be left unordered. Specifically, define the binary relation $\succeq_{P_{f}}$ on $A_{P_{f}}$ obtained from $P_{f}$ as follows: for all $S, S^{\prime} \in A_{P_{f}}$,

$$
S \succeq_{P_{f}} S^{\prime} \text { if and only if } S=C h\left(S \cup S^{\prime}, P_{f}\right) \text {. }
$$

Again, the binary relation $\succeq_{P_{f}}$ on $A_{P_{f}}$ leaves as unordered (i) all sets in $2^{W}$ that are not the choice of themselves and (ii) those pairs of sets in $A_{P_{f}}$ whose union contains a set that is preferred to each of the two sets. ${ }^{12}$ Martínez, Massó, Neme, and Oviedo (2008) show that if $P_{f}$ is substitutable then $\succeq_{P_{f}}$ is a partial order on $A_{P_{f}}$ and $\left(A_{P_{f}}, \succeq_{P_{f}}\right)$ is a semilattice; namely, for every $S, S^{\prime} \in A_{P_{f}}, l u b_{\succeq_{P_{f}}}\left\{S, S^{\prime}\right\} \in A_{P_{f}}$ (where, given a family of subsets $\mathcal{T}, l u b_{\succeq_{P_{f}}} \mathcal{T}$ means the least upper bound of $\mathcal{T}$ ). Example 2 below shows that if $P_{f}$ is not substitutable then the binary relation $\succeq_{P_{f}}$ may not even be transitive.

Example 2 Let $W=\left\{w_{1}, w_{2}, w_{3}, w_{4}, w_{5}\right\}$ be the set of workers and let $f$ be a firm. Consider the preference relation

$$
P_{f}:\left\{w_{1}, w_{5}\right\},\left\{w_{1}, w_{2}\right\},\left\{w_{3}, w_{4}\right\},\left\{w_{4}, w_{5}\right\},\{\varnothing\}
$$

where we only list acceptable subsets of workers in decreasing order of preference. Observe that $P_{f}$ is not substitutable since $w_{5} \in C h\left(W, P_{f}\right)$ and $w_{5} \notin C h\left(W \backslash\left\{w_{1}\right\}, P_{f}\right)=\left\{w_{3}, w_{4}\right\}$. Moreover, the family of individually rational subsets of workers relative to $P_{f}$ is $A_{P_{f}}=$ $\left\{\left\{w_{1}, w_{5}\right\},\left\{w_{1}, w_{2}\right\},\left\{w_{3}, w_{4}\right\},\left\{w_{4}, w_{5}\right\},\{\varnothing\}\right\}$ and

$$
\begin{aligned}
& \left\{w_{1}, w_{2}\right\}=C h\left(\left\{w_{1}, w_{2}\right\} \cup\left\{w_{3}, w_{4}\right\}, P_{f}\right), \\
& \left\{w_{3}, w_{4}\right\}=C h\left(\left\{w_{3}, w_{4}\right\} \cup\left\{w_{4}, w_{5}\right\}, P_{f}\right), \text { and } \\
& \left\{w_{1}, w_{5}\right\}=C h\left(\left\{w_{1}, w_{2}\right\} \cup\left\{w_{4}, w_{5}\right\}, P_{f}\right) .
\end{aligned}
$$

\footnotetext{
${ }^{12}$ Blair (1988) was the first to use this binary relation when showing that the set of stable matchings with multiple partners has a lattice structure.
} 
Hence, $\left\{w_{1}, w_{2}\right\} \succeq_{P_{f}}\left\{w_{3}, w_{4}\right\} \succeq_{P_{f}}\left\{w_{4}, w_{5}\right\}$ but $\left\{w_{1}, w_{2}\right\} \nsucceq_{P_{f}}\left\{w_{4}, w_{5}\right\}$. Thus, the binary relation $\succeq_{P_{f}}$ is not transitive and $\left(A_{P_{f}}, \succeq_{P_{f}}\right)$ is not a semilattice.

\subsection{Preliminary Results}

Example 2 shows that the binary relation $\succeq_{P_{f}}$ may be incomplete on $A_{P_{f}}$ (both $\left\{w_{1}, w_{2}\right\} \nsucceq_{P_{f}}$ $\left\{w_{4}, w_{5}\right\}$ and $\left\{w_{4}, w_{5}\right\} \nsucceq_{P_{f}}\left\{w_{1}, w_{2}\right\}$ hold) and that it may not inherit the transitivity of $P_{f}$. Nevertheless, Remark 1 and Proposition 1 below establish that the binary relation $\succeq_{P_{f}}$ inherit some other properties from the preference relation $P_{f}$.

Remark 1 Let $S, S^{\prime} \in A_{P_{f}}$ be such that $S \succeq_{P_{f}} S^{\prime}$. Then, $S R_{f} S^{\prime}$.

Proposition 1 Let $P_{f}$ be a preference relation over $2^{W}$. Then, the binary relation $\succeq_{P_{f}}$ on $A_{P_{f}}$ is reflexive, antisymmetric, acyclic, and has a maximal element on $A_{P_{f}}$.

Proof Let $S \in A_{P_{f}}$. Since $S=C h\left(S, P_{f}\right)=C h\left(S \cup S, P_{f}\right), S \succeq_{P_{f}} S$. Thus, $\succeq_{P_{f}}$ is reflexive.

Let $S, S^{\prime} \in A_{P_{f}}$ and suppose $S \succeq_{P_{f}} S^{\prime}$ and $S^{\prime} \succeq_{P_{f}} S$. Since $S=C h\left(S \cup S^{\prime}, P_{f}\right)=S^{\prime}$, $S=S^{\prime}$. Thus, $\succeq_{P_{f}}$ is antisymmetric.

Let $S_{1}, \ldots, S_{k} \in A_{P_{f}}$ be such that

$$
S_{1} \neq S_{k}
$$

and suppose $S_{1} \succeq_{P_{f}} \cdots \succeq_{P_{f}} S_{k}$ and $S_{k} \succeq_{P_{f}} S_{1}$. By Remark 1,

$$
S_{k} R_{f} S_{1}
$$

and $S_{1} R_{f} \cdots R_{f} S_{k}$. By transitivity of $R_{f}, S_{1} R_{f} S_{k}$. By (3), $S_{1} P_{f} S_{k}$. This contradicts (4). Thus, $\succeq_{P_{f}}$ is acyclic.

Let $S=C h\left(W, P_{f}\right)$ be the most preferred subset of workers according to $P_{f}$; i.e., for all $S^{\prime} \in 2^{W} \backslash S, S P_{f} S^{\prime}$. Thus, $S=C h\left(S, P_{f}\right)$ and $S \in A_{P_{f}}$. By definition of $S$,

$$
S=C h\left(S \cup S^{\prime}, P_{f}\right)
$$

for all $S^{\prime} \in 2^{W}$. In particular, if $S^{\prime} \in A_{P_{f}}$ then, (5) holds. Hence, $S \succeq_{P_{f}} S^{\prime}$ for all $S^{\prime} \in A_{P_{f}}$. Since $\succeq_{P_{f}}$ is antisymmetric, $S \succ_{P_{f}} S^{\prime}$ for all $S^{\prime} \in A_{P_{f}} \backslash S$. Thus, $\succeq_{P_{f}}$ has a maximal element on $A_{P_{f}}$. 


\section{The Invariance Result}

Theorem 1 below gives a simple procedure to partition the set of firm $f$ 's preference relations into equivalence classes where each class contains exactly those preference relations for which the set of Core matchings is invariant regardless of the other agents' preference relations. Theorem 1 says that an equivalence class is composed of all firm $f$ 's preference relations for which the binary relation obtained from them coincide.

Theorem 1 Let $P_{f}$ and $P_{f}^{\prime}$ be two preference relations over $2^{W}$. Then,

$$
\succeq_{P_{f}}=\succeq_{P_{f}^{\prime}} \text { if and only if } C\left(P_{f}, P_{-f}\right)=C\left(P_{f}^{\prime}, P_{-f}\right) \text { for all } P_{-f} \text {. }
$$

Proof $(\Rightarrow)$ Let $P_{f}$ and $P_{f}^{\prime}$ be two preference relations such that $\succeq_{P_{f}}=\succeq_{P_{f}^{\prime}}$. Thus, $A_{P_{f}}=A_{P_{f}^{\prime}}$. Assume there exist $P_{-f}$ and $\mu$ such that $\mu \in C\left(P_{f}, P_{-f}\right) \backslash C\left(P_{f}^{\prime}, P_{-f}\right)$. Since $A_{P_{f}}=A_{P_{f}^{\prime}}$ and $\mu \in C\left(P_{f}, P_{-f}\right), \mu(f) \in A_{P_{f}}=A_{P_{f}^{\prime}} ;$ i.e., $\mu(f)=C h\left(\mu(f), P_{f}^{\prime}\right)$. Thus,

$$
\mu \in I R\left(P_{f}, P_{-f}\right) \cap I R\left(P_{f}^{\prime}, P_{-f}\right) .
$$

Define $P^{\prime}=\left(P_{f}^{\prime}, P_{-f}\right)$ and let $\left(F^{\prime}, W^{\prime}, \mu^{\prime}\right)$ be a block of $\mu$ at $P^{\prime}$. By $(6)$, there exist $f^{\prime} \in F^{\prime}$ and $S^{\prime} \subseteq W^{\prime}$ such that $\mu^{\prime}\left(f^{\prime}\right)=S^{\prime}$ and for all $v \in\left\{f^{\prime}\right\} \cup S^{\prime}$,

$$
\mu^{\prime}(v) R_{v}^{\prime} \mu(v)
$$

and there exists $\bar{v} \in\left\{f^{\prime}\right\} \cup S^{\prime}$ such that

$$
\mu^{\prime}(\bar{v}) P_{\bar{v}}^{\prime} \mu(\bar{v})
$$

If $f^{\prime} \neq f$, by $(7)$ and $(8), \mu^{\prime}(v) R_{v} \mu(v)$ and $\mu^{\prime}(\bar{v}) P_{\bar{v}} \mu(\bar{v})$. Hence $\left(\left\{f^{\prime}\right\}, S^{\prime}, \mu^{\prime}\right)$ blocks $\mu$ at $\left(P_{f}, P_{-f}\right)$. This contradicts the hypothesis that $\mu \in C\left(P_{f}, P_{-f}\right)$. Thus, $f^{\prime}=f$. Hence $\left(\{f\}, \mu^{\prime}(f), \mu^{\prime}\right)$ blocks $\mu$ at $P^{\prime}$. If $\mu^{\prime}(f) \neq C h\left(\mu^{\prime}(f), P_{f}^{\prime}\right)$ then $\left(\{f\}, C h\left(\mu^{\prime}(f), P_{f}^{\prime}\right), \mu^{\prime \prime}\right)$ also blocks $\mu$ at $P$, where $\mu^{\prime \prime}$ is such that $\mu^{\prime \prime}(f)=C h\left(\mu^{\prime}(f), P_{f}^{\prime}\right)$ and $\mu^{\prime \prime}(\hat{f})=\varnothing$ for all $\hat{f} \neq f$. Hence, and since $A_{P_{f}}=A_{P_{f}^{\prime}}$, we can assume without loss of generality that $\mu^{\prime}(f)=C h\left(\mu^{\prime}(f), P_{f}^{\prime}\right)=C h\left(\mu^{\prime}(f), P_{f}\right)$. Since $\mu \in C\left(P_{f}, P_{-f}\right)$,

$$
\mu(f) P_{f} \mu^{\prime}(f) \text { and } \mu^{\prime}(f) P_{f}^{\prime} \mu(f) .
$$

Thus,

$$
\mu^{\prime}(f) \nsucceq_{P_{f}} \mu(f) \text { and } \mu(f) \nsucceq_{P_{f}^{\prime}} \mu^{\prime}(f) \text {. }
$$


By the hypothesis that $\succeq_{P_{f}}=\succeq_{P_{f}^{\prime}}$,

$$
\mu^{\prime}(f) \nsucceq_{P_{f}^{\prime}} \mu(f) \text { and } \mu(f) \nsucceq_{P_{f}} \mu^{\prime}(f) \text {. }
$$

Consider any matching $\hat{\mu}$ with the property that $\hat{\mu}(f)=C h\left(\mu(f) \cup \mu^{\prime}(f), P_{f}\right)$. We now show that, $\left(\{f\}, C h\left(\mu(f) \cup \mu^{\prime}(f), P_{f}\right), \hat{\mu}\right)$ blocks $\mu$ at $\left(P_{f}, P_{-f}\right)$. Since $(10)$ we have that $\hat{\mu}(f) P_{f} \mu(f)$. Let $w \in \hat{\mu}(f)$. Either $w \in \mu(f)$, in which case $w \in S^{\prime}$ and $\hat{\mu}(w) R_{w} \mu(w)$ since $\hat{\mu}(w)=\mu(w)$ or else, $w \in \mu^{\prime}(f)$, in which case $\hat{\mu}(w)=f=\mu^{\prime}(w) R_{w} \mu(w)$ by (7) and $R_{w}=R_{w}^{\prime}$. This contradicts the hypothesis that $\mu \in C\left(P_{f}, P_{-f}\right)$.

$(\Leftarrow)$ Let $P_{f}$ and $P_{f}^{\prime}$ be such that $C\left(P_{f}, P_{-f}\right)=C\left(P_{f}^{\prime}, P_{-f}\right)$ for all $P_{-f}$. We first show that $A_{P_{f}}=A_{P_{f}^{\prime}}$. Assume $S \in A_{P_{f}}$. We want to show that $S \in A_{P_{f}^{\prime}}$ (by symmetry, this will suffice). Consider the following subprofile $P_{-f}$ : for all $w \in S$, all $w^{\prime} \notin S$, and all $\hat{f} \neq f$,

$$
\begin{array}{lll}
\frac{P_{w}}{f} & \frac{P_{w^{\prime}}}{\varnothing} & \frac{P_{\hat{f}}}{\varnothing} \\
\varnothing . & &
\end{array}
$$

The unique core matching at $\left(P_{f}, P_{-f}\right)$ is $\mu$, where $\mu(f)=S$ and $\mu(\hat{f})=\varnothing$ for all $\hat{f} \neq f$ (obviously, $\mu\left(w^{\prime}\right)=\varnothing$ for all $w^{\prime} \notin S$ ). By hypothesis, $C\left(P_{f}, P_{-f}\right)=C\left(P_{f}^{\prime}, P_{-f}\right)$. Hence, $\mu$ is individually rational at $\left(P_{f}^{\prime}, P_{-f}\right)$. Thus, $S \in A_{P_{f}^{\prime}}$. To show that $\succeq_{P_{f}}=\succeq_{P_{f}^{\prime}}$ assume $S_{1}, S_{2} \in A_{P_{f}}=A_{P_{f}^{\prime}}$ are such that $S_{1} \succeq_{P_{f}} S_{2}$, but $S_{1} \succeq_{P_{f}^{\prime}} S_{2}$. Then,

$$
S_{1} \neq C h\left(S_{1} \cup S_{2}, P_{f}^{\prime}\right)
$$

Consider the following preference profile $P_{-f}$ : for all $w \in S_{1} \cup S_{2}$, all $w^{\prime} \notin S_{1} \cup S_{2}$, and all $\hat{f} \neq f$,

$$
\begin{array}{lll}
\frac{P_{w}}{f} & \frac{P_{w^{\prime}}}{\varnothing} & \frac{P_{\hat{f}}}{\varnothing} \\
\varnothing . & &
\end{array}
$$

Let $\mu$ be the matching where $\mu(f)=S_{1}, \mu(\hat{f})=\varnothing$ for all $\hat{f} \neq f$, and $\mu\left(w^{\prime}\right)=\varnothing$ for all $w^{\prime} \notin$ $S_{1}$. Since $S_{1} \succeq_{P_{f}} S_{2}, S_{1}=C h\left(S_{1} \cup S_{2}, P_{f}\right)$. It is easy to check that $\mu \in C\left(P_{f}, P_{-f}\right)$. Since $S_{1} \neq C h\left(S_{1} \cup S_{2}, P_{f}^{\prime}\right), C h\left(S_{1} \cup S_{2}, P_{f}^{\prime}\right) P_{f}^{\prime} S_{1}$. Thus, $\left(\{f\}, C h\left(S_{1} \cup S_{2}, P_{f}^{\prime}\right), \mu^{\prime}\right)$, where $\mu^{\prime}$ is any matching such that $\mu^{\prime}(f)=C h\left(S_{1} \cup S_{2}, P_{f}^{\prime}\right)$, blocks $\mu$ at $\left(P_{f}^{\prime}, P_{-f}\right)$ since

$$
\mu^{\prime}(f) P_{f}^{\prime} \mu(f)=S_{1}
$$


and for all $w \in C h\left(S_{1} \cup S_{2}, P_{f}^{\prime}\right)$,

$$
f=\mu^{\prime}(w) R_{w} \mu(w)
$$

Hence, $\mu \notin C\left(P_{f}^{\prime}, P_{-f}\right)$. This contradicts the hypothesis that $C\left(P_{f}, P_{-f}\right)=C\left(P_{f}^{\prime}, P_{-f}\right)$.

An alternative way of describing Theorem 1 in terms of the Core correspondence is as follows. For a firm $f$ and its preference relation $P_{f}$ denote by $T_{f, P_{f}}$ the Core mapping that takes as arguments all subprofiles of preferences $P_{-f}$ of workers and remaining firms and such that $T_{f, P_{f}}\left(P_{-f}\right)=C\left(P_{f}, P_{-f}\right)$. Theorem 1 partitions the set of preference relations of firm $f$ into equivalence classes such that all preference relations in a class have the same Core mapping.

Finally, Theorem 1 has the following implication. Consider two preference profiles with the property that workers' preference relations (on acceptable firms) are the same and for each firm the two preference relations (in the two profiles) have the same binary relation. Then, the two profiles have the same Core.

\section{The Minimality Result}

An implication of Theorem 1 is that an incomplete binary relation can be used as the representative of each equivalence class of all preference relations of a firm that leave the Core invariant. In general, the amount of information contained in the incomplete binary relation is substantially smaller than the one contained in any of its associated preference relations. However, this binary relation still contains redundant information (some pairs of subsets of workers are unnecessarily ordered) since the same equivalence class could be recovered by extending appropriately a strictly weaker binary relation. Example 3 below illustrates this fact and how we will proceed.

Example 3 Let $W=\left\{w_{1}, w_{2}, w_{3}\right\}$ be the set of workers and let $f$ be a firm. Consider the preference relation $P_{f}$ over $2^{W}$

$$
\left\{w_{1}, w_{2}, w_{3}\right\} P_{f}\left\{w_{1}, w_{2}\right\} P_{f}\left\{w_{1}, w_{3}\right\} P_{f}\left\{w_{2}, w_{3}\right\} P_{f}\left\{w_{1}\right\} P_{f}\left\{w_{2}\right\} P_{f}\left\{w_{3}\right\} P_{f}\{\varnothing\} .
$$

Observe that $A_{P_{f}}=2^{W}$. Obviously, $S \succeq_{P_{f}} S$ for all $S \in A_{P_{f}}$. In addition, $\succeq_{P_{f}}$ consists of 
the following orderings:

$$
\begin{array}{lll}
\left\{w_{1}, w_{2}, w_{3}\right\} \succeq_{P_{f}}\left\{w_{1}, w_{2}\right\} & \left\{w_{1}, w_{2}\right\} \succeq_{P_{f}}\left\{w_{1}\right\} & \left\{w_{1}\right\} \succeq_{P_{f}}\{\varnothing\} \\
\left\{w_{1}, w_{2}, w_{3}\right\} \succeq_{P_{f}}\left\{w_{1}, w_{3}\right\} & \left\{w_{1}, w_{2}\right\} \succeq_{P_{f}}\left\{w_{2}\right\} & \left\{w_{2}\right\} \succeq_{P_{f}}\{\varnothing\} \\
\left\{w_{1}, w_{2}, w_{3}\right\} \succeq_{P_{f}}\left\{w_{2}, w_{3}\right\} & \left\{w_{1}, w_{2}\right\} \succeq_{P_{f}}\{\varnothing\} & \left\{w_{3}\right\} \succeq_{P_{f}}\{\varnothing\} . \\
\left\{w_{1}, w_{2}, w_{3}\right\} \succeq_{P_{f}}\left\{w_{1}\right\} & \left\{w_{1}, w_{3}\right\} \succeq_{P_{f}}\left\{w_{1}\right\} & \\
\left\{w_{1}, w_{2}, w_{3}\right\} \succeq_{P_{f}}\left\{w_{2}\right\} & \left\{w_{1}, w_{3}\right\} \succeq_{P_{f}}\left\{w_{3}\right\} & \\
\left\{w_{1}, w_{2}, w_{3}\right\} \succeq_{P_{f}}\left\{w_{3}\right\} & \left\{w_{1}, w_{3}\right\} \succeq_{P_{f}}\{\varnothing\} & \\
\left\{w_{1}, w_{2}, w_{3}\right\} \succeq_{P_{f}}\{\varnothing\} & \left\{w_{2}, w_{3}\right\} \succeq_{P_{f}}\left\{w_{2}\right\} & \\
& \left\{w_{2}, w_{3}\right\} \succeq_{P_{f}}\left\{w_{3}\right\} & \\
& \left\{w_{2}, w_{3}\right\} \succeq_{P_{f}}\{\varnothing\} &
\end{array}
$$

Note that $\left\{w_{1}, w_{2}\right\} P_{f}\left\{w_{1}, w_{3}\right\},\left\{w_{1}, w_{2}\right\} P_{f}\left\{w_{2}, w_{3}\right\}$, and $\left\{w_{1}, w_{3}\right\} P_{f}\left\{w_{2}, w_{3}\right\}$ but $\left\{w_{1}, w_{2}\right\} \nsucceq P_{f}$ $\left\{w_{1}, w_{3}\right\},\left\{w_{1}, w_{2}\right\} \nsucceq_{P_{f}}\left\{w_{2}, w_{3}\right\}$, and $\left\{w_{1}, w_{3}\right\} \nsucceq_{P_{f}}\left\{w_{2}, w_{3}\right\}$ because $\left\{w_{1}, w_{2}, w_{3}\right\}=$ $C h\left(\left\{w_{1}, w_{2}\right\} \cup\left\{w_{1}, w_{3}\right\}, P_{f}\right)=C h\left(\left\{w_{1}, w_{2}\right\} \cup\left\{w_{2}, w_{3}\right\}, P_{f}\right)=C h\left(\left\{w_{1}, w_{3}\right\} \cup\left\{w_{2}, w_{3}\right\}, P_{f}\right)$. From the point of view of the Core, the only relevant information contained in $P_{f}$ (together with the fact that $A_{P_{f}}=2^{W}$ ) is that the best subset of workers is $W$ itself (this is true as long as we extend the binary relation by making sure that if one set of workers is strictly contained in another set then, the larger set is strictly preferred to the smaller one in the extension). The relative orderings among subsets of cardinality two and the relative orderings among subsets of cardinality one are irrelevant for the set of Core matchings. For instance, in this case the three preference relations $P_{f}^{\prime}, P_{f}^{\prime \prime}$, and $P_{f}^{\prime \prime \prime}$ defined by

$$
\begin{aligned}
& \left\{w_{1}, w_{2}, w_{3}\right\} P_{f}^{\prime}\left\{w_{1}, w_{2}\right\} P_{f}^{\prime}\left\{w_{2}, w_{3}\right\} P_{f}^{\prime}\left\{w_{1}, w_{3}\right\} P_{f}^{\prime}\left\{w_{1}\right\} P_{f}^{\prime}\left\{w_{2}\right\} P_{f}^{\prime}\left\{w_{3}\right\} P_{f}^{\prime}\{\varnothing\} \\
& \left\{w_{1}, w_{2}, w_{3}\right\} P_{f}^{\prime \prime}\left\{w_{1}, w_{2}\right\} P_{f}^{\prime \prime}\left\{w_{1}, w_{3}\right\} P_{f}^{\prime \prime}\left\{w_{2}, w_{3}\right\} P_{f}^{\prime \prime}\left\{w_{2}\right\} P_{f}^{\prime \prime}\left\{w_{1}\right\} P_{f}^{\prime \prime}\left\{w_{3}\right\} P_{f}^{\prime \prime}\{\varnothing\} \\
& \left\{w_{1}, w_{2}, w_{3}\right\} P_{f}^{\prime \prime \prime}\left\{w_{1}, w_{2}\right\} P_{f}^{\prime \prime \prime}\left\{w_{2}, w_{3}\right\} P_{f}^{\prime \prime \prime}\left\{w_{1}, w_{3}\right\} P_{f}^{\prime \prime \prime}\left\{w_{2}\right\} P_{f}^{\prime \prime \prime}\left\{w_{1}\right\} P_{f}^{\prime \prime \prime}\left\{w_{3}\right\} P_{f}^{\prime \prime \prime}\{\varnothing\}
\end{aligned}
$$

have the property that $C\left(P_{f}, P_{-f}\right)=C\left(P_{f}^{\prime}, P_{-f}\right)=C\left(P_{f}^{\prime \prime}, P_{-f}\right)=C\left(P_{f}^{\prime \prime \prime}, P_{-f}\right)$ for any subprofile $P_{-f}$. Indeed, we will show that from the information conveyed by the fact that $A_{P_{f}}=2^{W}$ and the much weaker (and minimal) binary relation $\succeq_{P_{f}}^{m}=\{\varnothing\}$, where no pair of subsets of workers are related, we will be able to extract the class of (complete) preference relations on $2^{W}$ that leave the Core invariant. Observe that the number of pairs related by $\succeq_{P_{f}}$ (nineteen or twenty seven if we include those that follow from reflexivity) is much larger than the number of pairs related by $\succeq_{P_{f}}^{m}$ (none). But this is an extreme case. 
Consider now the preference relation $\hat{P}_{f}$ over $2^{W}$

$$
\left\{w_{1}, w_{2}\right\} \hat{P}_{f}\left\{w_{1}\right\} \hat{P}_{f}\left\{w_{2}\right\} \hat{P}_{f}\left\{w_{3}\right\} \hat{P}_{f}\{\varnothing\},
$$

where we only list acceptable partners. Then, $A_{\hat{P}_{f}}=\left\{\left\{w_{1}, w_{2}\right\},\left\{w_{1}\right\},\left\{w_{2}\right\},\left\{w_{3}\right\},\{\varnothing\}\right\}$ and $\left\{w_{1}, w_{2}\right\} \succeq_{\hat{P}_{f}}\left\{w_{1}\right\},\left\{w_{1}, w_{2}\right\} \succeq_{\hat{P}_{f}}\left\{w_{2}\right\},\left\{w_{1}, w_{2}\right\} \succeq_{\hat{P}_{f}}\left\{w_{3}\right\},\left\{w_{1}\right\} \succeq_{\hat{P}_{f}}\left\{w_{3}\right\},\left\{w_{2}\right\} \succeq_{\hat{P}_{f}}$ $\left\{w_{3}\right\}$, and for any $S \in A_{\hat{P}_{f}}, S \succeq_{\hat{P}_{f}} S$ and $S \succeq_{\hat{P}_{f}}\{\varnothing\}$. Observe first that $\left\{w_{1}\right\} \nsucceq_{\hat{P}_{f}}\left\{w_{2}\right\}$ and $\left\{w_{2}\right\} \nsucceq_{\hat{P}_{f}}\left\{w_{1}\right\}$, and that the orderings $\left\{w_{1}, w_{2}\right\} \succeq_{\hat{P}_{f}}\left\{w_{3}\right\}$ and $\left\{w_{1}, w_{2}\right\} \succeq_{\hat{P}_{f}}\{\varnothing\}$ could be recovered by transitivity (using either $\left\{w_{1}\right\}$ or $\left\{w_{2}\right\}$ as intermediate subset). In addition, the orderings $\left\{w_{1}, w_{2}\right\} \succeq_{\hat{P}_{f}}\left\{w_{1}\right\},\left\{w_{1}, w_{2}\right\} \succeq_{\hat{P}_{f}}\left\{w_{2}\right\}$ and for any $S \in A_{\hat{P}_{f}}, S \succeq_{\hat{P}_{f}} S$ and $S \succeq_{\hat{P}_{f}}\{\varnothing\}$ could also be recovered because they relate a set with one of its subsets. Thus, we could define a much weaker binary relation $\succeq_{\hat{P}_{f}}^{m}$ on $A_{\hat{P}_{f}}$ with only two elements: $\left\{w_{1}\right\} \succeq_{\hat{P}_{f}}^{m}\left\{w_{3}\right\}$ and $\left\{w_{2}\right\} \succeq_{\hat{P}_{f}}^{m}\left\{w_{3}\right\}$. Moreover, given $A_{\hat{P}_{f}}$ (this conveys a very important information), the two preference relations $\hat{P}_{f}$ (the one that we started with) and $\bar{P}_{f}$ over $2^{W}$ (again, we only list acceptable partners)

$$
\begin{aligned}
& \left\{w_{1}, w_{2}\right\} \hat{P}_{f}\left\{w_{1}\right\} \hat{P}_{f}\left\{w_{2}\right\} \hat{P}_{f}\left\{w_{3}\right\} \hat{P}_{f}\{\varnothing\} \\
& \left\{w_{1}, w_{2}\right\} \bar{P}_{f}\left\{w_{2}\right\} \bar{P}_{f}\left\{w_{1}\right\} \bar{P}_{f}\left\{w_{3}\right\} \bar{P}_{f}\{\varnothing\}
\end{aligned}
$$

can be obtained from $\succeq_{\hat{P}_{f}}^{m}$ as what we will call strict extensions. Our results will say that $C\left(\hat{P}_{f}, P_{-f}\right)=C\left(\bar{P}_{f}, P_{-f}\right)$ for all subprofiles $P_{-f}$. On the other hand, if we had left the two subsets $\left\{w_{1}\right\}$ and $\left\{w_{3}\right\}$ unordered we could have found two strict extensions $\check{P}_{f}$ and $\tilde{P}_{f}$ with the property that $C\left(\check{P}_{f}, P_{-f}\right) \neq C\left(\tilde{P}_{f}, P_{-f}\right)$ for some subprofile $P_{-f}$; in this sense the binary relation $\succeq_{\hat{P}_{f}}^{m}$ on $A_{\hat{P}_{f}}$ will be called minimal.

In the sequel we define a minimal binary relation that will declare as unordered (i) any two subsets of workers with the property that one is a strict subset of the other and (ii) any two subsets of workers whose relative ordering (for instance, $S \succeq_{P_{f}} S^{\prime \prime}$ ) could be obtained by transitivity (i.e., $S$ and $S^{\prime \prime}$ will be left unordered whenever there exists $S^{\prime} \in A_{P_{f}}$ such that $\left.S \succeq_{P_{f}} S^{\prime} \succeq_{P_{f}} S^{\prime \prime}\right)$.

In this section we identify the minimal binary relation (weaker than the one used as the representative of the class) with the following two properties: (i) all preference relations in the class can be obtained from this minimal binary relation by what we call a strict extension, and (ii) any strictly weaker binary relation has at least two strict extensions that belong to two different equivalence classes. 


\subsection{Transitive Closure}

To make the proof of Theorem 2 below simpler, we will now first enlarge $\succeq_{P_{f}}$ with its transitive closure $\succeq_{P_{f}}^{T}$ and then reduce it by identifying a minimal binary relation $\succeq_{P_{f}}^{m}$ so that $\succeq_{P_{f}}^{m} \subseteq \succeq_{P_{f}} \subseteq \succeq_{P_{f}}^{T}$.

Definition 5 Let $\succeq$ be an acyclic binary relation on $A$. The binary relation $\succeq^{T}$ on $A$ is the transitive closure of $\succeq$ if it is the smallest transitive binary relation on $A$ that contains $\succeq$.

Notice that the all-relation on $A \times A$ is transitive and contains all binary relations on $A$. The intersection of transitive binary relations on $A$ is again transitive; that is, given $\succeq$, $\succeq^{T}=\cap\left\{\succeq^{\prime} \subseteq A \times A \mid \succeq^{\prime} \supseteq \succeq\right.$ and $\succeq^{\prime}$ is transitive $\}$ is transitive. Finally, let $\succeq$ be an acyclic binary relation on $A$. Then, $\succeq^{T}=\cup_{n \in \mathbb{N}} \succeq^{n}$, where $S \succeq^{n} S^{\prime}$ if there exist $S_{1}, \ldots, S_{n} \in A$ such that $S=S_{1} \succeq \cdots \succeq S_{n}=S^{\prime}{ }^{13}$

Before proceeding we state and prove a Lemma that will be useful in the sequel.

Lemma 1 Let $P_{f}$ be a preference relation over $2^{W}$ and assume that $S_{1}, S_{2} \in A_{P_{f}}, S_{1} \succeq_{P_{f}}^{T}$ $S_{2}$, and $S_{1} \subseteq S_{2}$. Then, $S_{1}=S_{2}$.

Proof Since $S_{1} \subseteq S_{2}$ and $S_{2} \in A_{P_{f}}, C h\left(S_{1} \cup S_{2}, P_{f}\right)=C h\left(S_{2}, P_{f}\right)=S_{2}$. Hence, $S_{2} \succeq_{P_{f}}$ $S_{1}$ and $S_{2} \succeq_{P_{f}}^{T} S_{1}$. By hypothesis, $S_{1} \succeq_{P_{f}}^{T} S_{2}$. By Proposition $1, \succeq_{P_{f}}$ is antisymmetric. By its definition, $\succeq_{P_{f}}^{T}$ is antisymmetric as well. Thus, $S_{1}=S_{2}$.

\subsection{Minimal Binary Relation}

To identify the minimal binary relation associated to the preference relation $P_{f}$ of firm $f$ we proceed as follows. First, obtain $A_{P_{f}}$. Second, compute $\succeq_{P_{f}}$ and its transitive closure $\succeq_{P_{f}}^{T}$. Then, delete from $\succeq_{P_{f}}^{T}$ all ordered pairs of subsets of workers that (i) are related by inclusion and (ii) are related as the consequence of the transitivity of $\succeq_{P_{f}}^{T}$. Formally,

Definition 6 Let $P_{f}$ be a preference relation over $2^{W}$. The binary relation $\succeq \subseteq \succeq_{P_{f}}^{T}$ on $A_{P_{f}}$ is minimal if for all $S, S^{\prime} \in A_{P_{f}}$ such that $S \succeq_{P_{f}}^{T} S^{\prime}$ the following condition holds:

(mi) $S \succeq S^{\prime}$ if and only if $S \cap S^{\prime} \notin\left\{S, S^{\prime}\right\}$ and there does not exist $S^{\prime \prime} \in A_{P_{f}} \backslash\left\{S, S^{\prime}\right\}$ such that, $S \succeq_{P_{f}}^{T} S^{\prime \prime} \succeq_{P_{f}}^{T} S^{\prime}$.

\footnotetext{
${ }^{13}$ See Harzheim (2005).
} 
Given $P_{f}$, there is a unique minimal binary relation on $A_{P_{f}}$. Denote it by $\succeq_{P_{f}}^{m}$. Next lemma states that $\succeq_{P_{f}}^{m}$ is not only weaker than $\succeq_{P_{f}}^{T}$ but it is also weaker than the original $\succeq_{P_{f}}$.

Lemma 2 Let $P_{f}$ be a preference relation over $2^{W}$. Then, $\succeq_{P_{f}}^{m} \subseteq \succeq_{P_{f}}$.

Proof To obtain a contradiction assume $\succeq_{P_{f}}^{m} \nsubseteq_{\succeq_{P_{f}}}$; namely, there exist $S, S^{\prime} \in A_{P_{f}}$ such that $S \succeq_{P_{f}}^{m} S^{\prime}$ and $S \succeq_{P_{f}} S^{\prime}$. Since, by Proposition $1, \succeq_{P_{f}}$ is reflexive, $S \neq S^{\prime}$. Observe that $\succeq_{P_{f}}^{m} \subseteq \succeq_{P_{f}}^{T}$ and $S \succeq_{P_{f}}^{m} S^{\prime}$ imply $S \succeq_{P_{f}}^{T} S^{\prime}$. Since $S \succeq_{P_{f}} S^{\prime}$ and $S \succeq_{P_{f}}^{T} S^{\prime}$, by definition of $\succeq_{P_{f}}^{T}$ there exists $S_{1}, \ldots, S_{n} \in A_{P_{f}}$ such that $S \neq S_{1} \neq \cdots \neq S_{n} \neq S^{\prime}$ and $S \succeq_{P_{f}} S_{1} \succeq_{P_{f}} \cdots \succeq_{P_{f}} S_{n} \succeq_{P_{f}} S^{\prime}$. Since $\succeq_{P_{f}}^{T}$ is transitive by definition, this implies that $S \succeq_{P_{f}}^{T} S_{1} \succeq_{P_{f}}^{T} S^{\prime}$. By (mi) in Definition $6, S \succeq_{P_{f}}^{m} S^{\prime}$, a contradiction.

Alternatively, we could directly define $\succeq_{P_{f}}^{m} \subseteq \succeq_{P_{f}}$ replacing condition (mi) in Definition 6 above by

(mi') $S \succeq S^{\prime}$ if and only if $S \cap S^{\prime} \notin\left\{S, S^{\prime}\right\}$ and there does not exist $S^{\prime \prime} \in A_{P_{f}} \backslash\left\{S, S^{\prime}\right\}$ such that, $S \succeq_{P_{f}} S^{\prime \prime} \succeq_{P_{f}} S^{\prime}$.

However, the arguments would become more involved since instead of using the transitivity of $\succeq_{P_{f}}^{T}$ we should use the acyclicity of $\succeq_{P_{f}}$ by identifying (and working with) sequences $S_{1} \succeq_{P_{f}} \cdots \succeq_{P_{f}} S_{k}$ with the property that $S_{k} \succeq_{P_{f}} S_{1}$.

\subsection{Strict Extension}

We next give a procedure to obtain from the minimal binary relation all preference relations that would generate it. The procedure consists of completing the acyclic minimal binary relation by declaring a set in the family to be (strictly) preferred to all its subsets and if a set is not in the family of individually rational subsets of workers then it must have a strict subset that belongs to the family and is strictly preferred to it. Formally,

Definition $7 \quad$ Let $\succeq$ be an acyclic binary relation on $A \subset 2^{W}$ with $\varnothing \in A$. The (strict) preference relation $\bar{P}_{f}$ over $2^{W}$ is a strict extension of $\succeq$ if for all $S^{\prime}, S^{\prime \prime} \in A$ such that $S^{\prime} \neq S^{\prime \prime}$ :

(se.1) if $S^{\prime} \succ S^{\prime \prime}$ then, $S^{\prime} \bar{P}_{f} S^{\prime \prime}$;

(se.2) if $S^{\prime \prime} \varsubsetneqq S^{\prime}$ then, $S^{\prime} \bar{P}_{f} S^{\prime \prime}$; and 
(se.3) if $S \notin A$ then, there exists $\hat{S} \in A$ such that $\hat{S} \varsubsetneqq S$ and $\hat{S} \bar{P}_{f} S$.

Definition 7 can be seen as a set of instructions on how to extend an acyclic binary relation on $A$ to a preference relation over $2^{W}$. First, it preserves all the ordered pairs (this corresponds to the standard notion of an extension used by Szpilrajn (1930)). Second, a set is preferred to all its subsets. Third, if a set is not in $A$ then, we have freedom on how to order it but the set has to be worse than one of its subsets (perhaps the empty set). Finally, all the remaining pairs that are not ordered by the acyclic binary relation can be freely ordered by the preference relation (this is one of the reasons of why in general there are many strict extensions of an acyclic binary relation). Before proceeding, we state and prove two results: Lemma 3 will be useful in the proof of Theorem 2 below and Lemma 4 states that indeed $P_{f}$ is obtained as a strict extension of $\succeq_{P_{f}}^{m}$.

Lemma 3 Let $P_{f}$ be a preference relation over $2^{W}$. Suppose $\bar{P}_{f}$ is a strict extension of $\succeq_{P_{f}}^{m}$. Furthermore, assume $S, S^{\prime} \in A_{P_{f}}, S \neq S^{\prime}$ and $S \succeq_{P_{f}}^{T} S^{\prime}$. Then, $S \bar{P}_{f} S^{\prime}$.

Proof If $S \succeq_{P_{f}}^{m} S^{\prime}$ then, by (se.1) in Definition 7, $S \bar{P}_{f} S^{\prime}$.

Assume $S \nsucceq_{P_{f}}^{m} S^{\prime}$. By Lemma 1 , and since $S \succeq_{P_{f}}^{T} S^{\prime}$ and $S \neq S^{\prime}, S \nsubseteq S^{\prime}$. Thus, either $S \supseteq S^{\prime}$ or there exists $S_{1} \in A_{P_{f}}$ such that $S \succeq_{P_{f}}^{T} S_{1} \succeq_{P_{f}}^{T} S^{\prime}$. If $S \supseteq S^{\prime}$ then, by (se.2) in Definition $7, S \bar{P}_{f} S^{\prime}$.

Assume there exists $S_{1} \in A_{P_{f}}$ such that $S_{1} \neq S, S^{\prime}$ and $S \succeq_{P_{f}}^{T} S_{1} \succeq_{P_{f}}^{T} S^{\prime}$, We assume without lost of generality that $S_{1}$ is maximal with respect to $\succeq_{P_{f}}^{T}$; i.e., there does not exist $\hat{S} \in A, \hat{S} \neq S_{1}, S, S^{\prime}$ such that $S \succeq_{P_{f}}^{T} \hat{S} \succeq_{P_{f}}^{T} S_{1} \succeq_{P_{f}}^{T} S^{\prime}$. Observe that by Proposition 1, $\succeq_{P_{f}}$ is acyclic and hence, by Lemma $2, \succeq_{P_{f}}^{m}$ is also acyclic. Thus, this maximal set $S_{1}$ does exist.

Assume $S \nsucceq_{P_{f}}^{m} S_{1}$. Then, if there would exist $\hat{S} \in A_{P_{f}}$ such that $S \succeq_{P_{f}}^{T} \hat{S} \succeq_{P_{f}}^{T} S_{1}$ then, $S \succeq_{P_{f}}^{T} \hat{S} \succeq_{P_{f}}^{T} S_{1} \succeq_{P_{f}}^{T} S^{\prime}$, contradicting the maximality of $S_{1}$. Because $S \succeq_{P_{f}}^{T} S_{1}$, by (mi) in Definition 6, either $S \supseteq S_{1}$ or $S_{1} \supseteq S$. Note that if $S_{1} \supseteq S$, and since $S \succeq_{P_{f}}^{T} S_{1}$, Lemma 1 implies $S=S^{\prime}$. This contradicts $S \Varangle_{P_{f}}^{m} S_{1}$ because, by Proposition 1, $\succeq_{P_{f}}$ is reflexive. Hence, and since $\succeq_{P_{f}}^{m} \subseteq \succeq_{P_{f}}^{T}, \succeq_{P_{f}}^{m}$ is reflexive as well. Thus, $S \supseteq S_{1}$. By (se.2) in Definition 7, any strict extension $\bar{P}_{f}$ of $\succeq_{P_{f}}^{m}$ satisfies $S \bar{P}_{f} S_{1}$.

Assume $S \succeq_{P_{f}}^{m} S_{1}$. By (se.1) in Definition 7, any strict extension $\bar{P}_{f}$ of $\succeq_{P_{f}}^{m}$ satisfies $S \bar{P}_{f} S_{1}$. 
We have already shown that $S \bar{P}_{f} S_{1}$ and $S_{1} \succeq_{P_{f}}^{T} S^{\prime}$. If $S_{1} \succeq_{P_{f}}^{m} S^{\prime}$, by (se.1) in Definition 7, $S_{1} \bar{P}_{f} S^{\prime}$, in which case, $S \bar{P}_{f} S^{\prime}$. If $S_{1} \nsucceq P_{P_{f}}^{m} S^{\prime}$, we repeat the argument above replacing the former role of $S$ by $S_{1}$. Since $A_{P_{f}}$ is finite, there exists a finite sequence $\left\{S_{1}, \ldots, S_{k}\right\}$ such that $S \bar{P}_{f} S_{1} \bar{P}_{f} \ldots \bar{P}_{f} S^{k} \bar{P}_{f} S^{\prime}$. By transitivity of $\bar{P}_{f}, S \bar{P}_{f} S^{\prime}$.

Lemma 4 Let $P_{f}$ be a preference relation over $2^{W}$. Then, $P_{f}$ is a strict extension of the minimal binary relation $\succeq_{P_{f}}^{m}$.

Proof We consider separately the three cases in Definition 7. For the first two, let $S_{1}, S_{2} \in A_{P_{f}}$ be such that $S_{1} \neq S_{2}$.

(se.1) Assume $S_{1} \succeq_{P_{f}}^{m} S_{2}$. By Lemma 2, $S_{1} \succeq_{P_{f}} S_{2} ;$ i.e., $S_{1}=C h\left(S_{1} \cup S_{2}, P_{f}\right)$. Since $S_{1} \neq S_{2}, S_{1} P_{f} S_{2}$.

(se.2) Assume $S_{2} \varsubsetneqq S_{1}$. To obtain a contradiction, assume $S_{2} P_{f} S_{1}$. Then, $S_{1} \neq C h\left(S_{1} \cup S_{2}, P_{f}\right)=$ $C h\left(S_{1}, P_{f}\right)$, where the equality follows because $S_{2} \varsubsetneqq S_{1}$. But this is a contradiction with $S_{1} \in A_{f}$.

(se.3) Let $S \notin A_{P_{f}}$. We want to show that there exists $\hat{S} \in A_{P_{f}}$ such that $\hat{S} \subsetneq S$ and $\hat{S} P_{f} S$. Note that $C h\left(S, P_{f}\right) \in A_{P_{f}}$ and $C h\left(S, P_{f}\right) \subseteq S$. Since $S \notin A_{P_{f}}, C h\left(S, P_{f}\right) P_{f} S$. Thus, set the desired $\hat{S}$ be equal to $C h\left(S, P_{f}\right)$. Then, $\hat{S} P_{f} S$.

\subsection{Results}

We are now ready to state and prove the two results of this section.

Theorem 2 Let $P_{f}$ be a preference relation over $2^{W}$ and assume $\bar{P}_{f}$ is a strict extension of the minimal binary relation $\succeq_{P_{f}}^{m}$. Then, $A_{\bar{P}_{f}}=A_{P_{f}}$ and $\succeq_{\bar{P}_{f}}=\succeq_{P_{f}}$.

Before proving Theorem 2 two remarks are in order. First, the statement of Theorem 2 implicitly contains the following procedure that we want to make explicit before we proceed to its proof. Given a preference relation $P_{f}$ over $2^{W}$, construct the family $A_{P_{f}}$ of individually rational subsets of workers relative to $P_{f}$. From $A_{P_{f}}$, obtain sequentially the binary relation $\succeq_{P_{f}}$ on $A_{P_{f}}$, its transitive closure $\succeq_{P_{f}}^{T}$, and the minimal binary relation $\succeq_{P_{f}}^{m}$. Then, take an arbitrary strict extension $\bar{P}_{f}$ of $\succeq_{P_{f}}^{m}$, construct the family $A_{\bar{P}_{f}}$ and its associated binary relation $\succeq_{\bar{P}_{f}}$. Theorem 2 says that $A_{P_{f}}=A_{\bar{P}_{f}}$ and $\succeq_{P_{f}}=\succeq_{\bar{P}_{f}}$ hold. Second, Theorem 2 implies (together with Theorem 1) that the minimal binary relation can be used as the representative of all preference relations that leave the set of Core matchings invariant; 
namely, $\succeq_{P_{f}}^{m}$ still contains all information needed to obtain the full equivalence class of preference relations as strict extensions of $\succeq_{P_{f}}^{m}$.

We now turn to prove Theorem 2.

Proof We first prove that $A_{\bar{P}_{f}}=A_{P_{f}}$. To show that $A_{\bar{P}_{f}} \subseteq A_{P_{f}}$, we will show that $S \notin A_{P_{f}}$ implies $S \notin A_{\bar{P}_{f}}$. Assume $S \notin A_{P_{f}}$. Hence, and since $\bar{P}_{f}$ is a strict extension of $\succeq_{P_{f}}^{m}$, by (se.3), there exists $\hat{S} \in A_{P_{f}}$ such that $\hat{S} \subsetneq S$ and $\hat{S} \bar{P}_{f} S$. Thus, $S \neq C h\left(S, \bar{P}_{f}\right)$ and $S \notin A_{\bar{P}_{f}}$.

To show that $A_{P_{f}} \subseteq A_{\bar{P}_{f}}$, assume $S \in A_{P_{f}} \backslash A_{\bar{P}_{f}}$. Hence,

$$
S=C h\left(S, P_{f}\right)
$$

and $S \neq C h\left(S, \bar{P}_{f}\right)$. Let $S^{\prime} \subsetneq S$ be such that $S^{\prime}=C h\left(S, \bar{P}_{f}\right)$. Obviously, $S^{\prime}=C h\left(S^{\prime}, \bar{P}_{f}\right)$ and

$$
S^{\prime} \bar{P}_{f} S
$$

Thus, $S^{\prime} \in A_{\bar{P}_{f}}$. Hence, and since we have already proved that $A_{\bar{P}_{f}} \subseteq A_{P_{f}}, S^{\prime} \in A_{P_{f}}$. Thus, $S^{\prime}=C h\left(S^{\prime}, P_{f}\right)$. By $S^{\prime} \varsubsetneqq S$ and (11), $S=C h\left(S \cup S^{\prime}, P_{f}\right)$. Hence, $S \succ_{P_{f}} S^{\prime}$. Thus, $S \succ_{P_{f}}^{T} S^{\prime}$. By Lemma 3, $S \bar{P}_{f} S^{\prime}$, a contradiction with (12).

Second, to prove $\succeq_{\bar{P}_{f}}=\succeq_{P_{f}}$ we will show that for all $S_{1}, S_{2} \in A_{\bar{P}_{f}}=A_{P_{f}}$,

$$
S_{1}=C h\left(S_{1} \cup S_{2}, \bar{P}_{f}\right) \text { if and only if } S_{1}=C h\left(S_{1} \cup S_{2}, P_{f}\right) .
$$

$\Longrightarrow)$ Assume $S_{1} \neq C h\left(S_{1} \cup S_{2}, P_{f}\right)$. Hence, $C h\left(S_{1} \cup S_{2}, P_{f}\right) \succ_{P_{f}} S_{1}$. By definition of $\succeq_{P_{f}}^{T}, C h\left(S_{1} \cup S_{2}, P_{f}\right) \succeq_{P_{f}}^{T} S_{1}$. By Lemma 3, Ch $\left(S_{1} \cup S_{2}, P_{f}\right) \bar{P}_{f} S_{1}$. Since $C h\left(S_{1} \cup S_{2}, P_{f}\right) \subseteq$ $S_{1} \cup S_{2}, S_{1} \neq C h\left(S_{1} \cup S_{2}, \bar{P}_{f}\right)$.

$\Longleftarrow)$ To obtain a contradiction, assume $S_{1}=C h\left(S_{1} \cup S_{2}, P_{f}\right)$ and $S_{1} \neq C h\left(S_{1} \cup S_{2}, \bar{P}_{f}\right)$. Then, $C h\left(S_{1} \cup S_{2}, \bar{P}_{f}\right) \succ_{\bar{P}_{f}} S_{1}$. Since $C h\left(S_{1} \cup S_{2}, \bar{P}_{f}\right) \subseteq S_{1} \cup S_{2}$ and $S_{1}=C h\left(S_{1} \cup S_{2}, P_{f}\right)$, $S_{1} \succeq_{P_{f}} C h\left(S_{1} \cup S_{2}, \bar{P}_{f}\right)$. By definition of $\succeq_{P_{f}}^{T}, S_{1} \succeq_{P_{f}}^{T} C h\left(S_{1} \cup S_{2}, \bar{P}_{f}\right)$. By Lemma 3, $S_{1} \bar{P}_{f} C h\left(S_{1} \cup S_{2}, \bar{P}_{f}\right)$, a contradiction with $C h\left(S_{1} \cup S_{2}, \bar{P}_{f}\right) \succ_{\bar{P}_{f}} S_{1}$.

Theorem 3 below states that $\succeq_{P_{f}}^{m}$ is indeed minimal in the sense that any strictly weaker binary relation generates, as one of its strict extensions, a preference relation of firm $f$ that belongs to a different equivalence class of the one to which $P_{f}$ belongs to and thus, with a different Core for some subprofile $P_{-f}$. 
Theorem 3 Let $P_{f}$ be a preference relation and assume $\succeq^{\prime} \beth_{\succeq_{P}}^{m}$. Then, there exists a strict extension $P_{f}^{\prime}$ of $\succeq^{\prime}$ such that $\succeq_{P_{f}^{\prime}} \neq \succeq_{P_{f}}$.

Proof Since $\succeq^{\prime} \Varangle_{P_{f}}^{m}$, there exist $S_{1}, S_{2} \in A_{P_{f}}$ such that $S_{1} \succeq_{P_{f}}^{m} S_{2}$, but $S_{1} \nsucceq^{\prime} S_{2}$. By (mi) in Definition 6, $S_{1} \succeq_{P_{f}}^{m} S_{2}$, implies that neither $S_{1} \supsetneq S_{2}$ nor $S_{2} \supsetneq S_{1}$. Let $P_{f}^{\prime}$ be a strict extension of $\succeq^{\prime}$ with the property that $S_{2} P_{f}^{\prime} S_{1}$. Observe that none of the hypothesis of conditions (se.1), (se.2), and (se.3) in Definition 7 hold; thus, there exists such extension $P_{f}^{\prime}$. Then, $S_{1} \neq C h\left(S_{1} \cup S_{2}, P_{f}^{\prime}\right)$. Hence, $\succeq_{P_{f}}^{m} \subseteq \succeq_{P_{f}}$ and $S_{1} \succeq_{P_{f}}^{m} S_{2}$ imply that, by Lemma $2, S_{1} \succeq_{P_{f}} S_{2}$; namely, $S_{1}=C h\left(S_{1} \cup S_{2}, P_{f}\right)$. Thus, $\succeq_{P_{f}} \neq \succeq_{P_{f}^{\prime}}$.

\section{Concluding Remark}

Our results give a systematic procedure to elicit from a given preference profile $P$ the minimal and indispensable information that is relevant for determining the set of Core matchings at $P$. Specifically, the main conclusions and implications of our results are the following.

First, consider a preference profile $P=\left(\left(P_{f}\right)_{f \in F},\left(P_{w}\right)_{w \in W}\right)$. For each $f \in F$, (a) construct $A_{P_{f}}$, the family of individually rational subsets of workers relative to $P_{f}$, by identifying those subsets of workers that are the choice of themselves; and (b) define the binary relation $\succeq_{P_{f}}$ on $A_{P_{f}}$ from $P_{f}$ by declaring, for each pair of subsets $S$ and $S^{\prime}$ in $A_{P_{f}}$, that $S$ is preferred (according to $\succeq_{P_{f}}$ ) to $S^{\prime}$ if and only if $S$ is the best subset of workers (according to $P_{f}$ ) among all subsets of the union of $S$ and $S^{\prime}$. All information needed to compute the set of Core matchings at preference profile $P=\left(\left(P_{f}\right)_{f \in F},\left(P_{w}\right)_{w \in W}\right)$ is contained in the profile $\succeq=\left(\left(\succeq_{P_{f}}\right)_{f \in F},\left(P_{w}\right)_{w \in W}\right)$. In addition, any preference profile $P^{\prime}=\left(\left(P_{f}^{\prime}\right)_{f \in F},\left(P_{w}\right)_{w \in W}\right)$ such that $\succeq_{P_{f}^{\prime}}=\succeq_{P_{f}}$ for all $f \in F$ has the property that the Core at $P^{\prime}$ coincides with the Core at $P$.

Second, this profile of incomplete binary relations is still too rich since they order pairs of subsets of workers that are related either by inclusion or by transitivity through an intermediate subset of workers. We obtain from $\succeq=\left(\left(\succeq_{P_{f}}\right)_{f \in F},\left(P_{w}\right)_{w \in W}\right)$ the profile $\succeq^{m}=$ $\left(\left(\succeq_{P_{f}}^{m}\right)_{f \in F},\left(P_{w}\right)_{w \in W}\right)$ with the properties that for each $f \in F,(1) \succeq_{P_{f}}^{m}$ is weaker than $\succeq_{P_{f}}$ in the sense that it is contained (as a subset of $A_{P_{f}} \times A_{P_{f}}$ ) in $\succeq_{P_{f}}$; (2) we can obtain the equivalence class of firm $f$ 's preference relations with the same set of Core matchings by 
strictly extending $\succeq_{P_{f}}^{m}$; and (3) $\succeq_{P_{f}}^{m}$ is the minimal binary relation for which properties (1) and (2) hold.

Thus, we can substantially reduce the amount of information required from firms about their preference relations when a direct revelation mechanism is applied to centralized, two-sided, many-to-one matchings markets. In particular, this is important when firms' preference relations are substitutable and the used mechanism is stable, as in many real centralized markets like entry-level professional labor markets.

\section{References}

[1] Blair, C. "The Lattice Structure of the Set of Stable Matchings with Multiple Partners," Mathematics of Operations Research 13, 619-628 (1988).

[2] Echenique, F. "What Matchings Can Be Stable? The Testable Implications of Matching Theory," Mathematics of Operations Research 33, 757-768 (2008).

[3] Echenique, F. and J .Oviedo. "Core Many-to-one Matching by Fixed Point Methods," Journal of Economic Theory 115, 358-376 (2004).

[4] Harzheim, E. Ordered Sets. Advances in Mathematics, Springer. New York (2005).

[5] Hatfield, J. and F. Kojima. "Matching with Contracts: Comment," American Economic Review 98, 1189-1194 (2008).

[6] Kelso, A. and V. Crawford. "Job Matchings, Coalition Formation, and Gross Substitutes," Econometrica 50, 1483-1504 (1982).

[7] Martínez, R., J. Massó, A. Neme, and J. Oviedo. "On the invariance of the set of stable matchings with respect to substitutable preference profiles," International Journal of Game Theory 36, 497-518 (2008).

[8] Niederle, M., A. Roth, and T. Sönmez. "Matching and Market Design," The New Palgrave Dictionary of Economics. 2nd Edition. Eds. Steven N. Durlauf and Lawrence E. Blume (2008). 
[9] Roth, A. and M. Sotomayor. Two-Sided Matching: A Study in Game-Theoretic Modeling and Analysis. Cambridge University Press. Cambridge (1990).

[10] Szpilrajn, E. "Sur l'extension de l'ordre partiel," Fundamenta Mathematicae 16, 386389 (1930). 